\title{
Analysis of junction capacitance characteristics of trench gate IGBT
}

\author{
Wang $\mathrm{Bo}^{1, *}$ \\ ${ }^{1}$ Wuhan Donghu University, CHINA
}

\begin{abstract}
Trench gate field termination IGBT represents the latest structure of insulated gate bipolar transistor (IGBT). Because the internal current of IGBT includes the charging and discharging current of gate capacitance and internal junction capacitance during switching transient, the influence of junction capacitance should be considered. The conductive channel of trench gate structure is different from that of planar gate structure, and the analysis method of junction capacitance using planar gate structure will inevitably bring some deviation. Based on the characteristics of trench gate structure, this paper analyzes the different expressions of internal gate-drain junction capacitance in two cases according to whether the base depletion layer can be widened to cover the trench gate, and finally carries out simulation and experimental verification.
\end{abstract}

\section{Introduction}

Insulated gate bipolar transistor (IGBT) is a composite device which combines the structure of field effect transistor and bipolar transistor, and absorbs the advantages of both. It has high input impedance, fast switching speed, low driving power, low saturation voltage, simple control circuit, large current bearing and so on. Widely used in new energy technology, energysaving technology and other fields, it has gradually become the leading device of medium and high-power power electronic devices ${ }^{[1-3]}$.

With the continuous improvement of design level and manufacturing level of power semiconductor devices, IGBT has also experienced the development process from planar gate type to trench gate type, and punch-through type to field termination type. The new generation of trench gate field termination IGBT has achieved a leap in power grade and comprehensive performance, and gradually occupied the dominant position of IGBT application. Due to the structural characteristics of trench gate field termination type, which is different from the traditional planar gate type, the two-dimensional distribution of carriers in the base region and the influence of injection and depletion layer broadening on junction capacitance are different. This method of directly adopting the existing model will inevitably bring great deviation ${ }^{[4-}$ 5]. In IGBT switching transient process, the change of external collector-emitter voltage will significantly affect the internal junction capacitance, and the charging and discharging current of junction capacitance constitutes a part of IGBT transient current. Therefore, accurate modeling of internal junction capacitance will significantly improve the accuracy of IGBT transient current, which has certain theoretical and engineering practical significance.

Based on the equivalent circuit analysis of internal junction capacitance of trench gate field-terminated IGBT, this paper discusses the calculation method of internal gate junction capacitance in two cases according to whether the base depletion layer can widen and cover the trench gate. The calculation method considers the influence of different collector-emitter voltages on the width of base depletion layer in IGBT transient process, which causes the change of internal gate junction capacitance of IGBT and improves the accuracy of IGBT transient model.

\section{Theoretical analysis}

\subsection{GBT junction capacitance characteristics}

IGBT, as a fully controlled power electronic device, can switch between on-state and off-state conveniently by charging and discharging the gate capacitance through an external driving circuit, so the gate capacitance directly affects the switching transient process of IGBT. The gate capacitance consists of gate-source capacitance, gate overlapping oxide capacitance and gate overlapping depletion capacitance, which varies with external voltage.

Schematic diagram of planar structure of trench gate and planar gate IGBT is shown in Figure 1, which can be regarded as BJT structure driven by MOSFET. The conductive channel of planar gate structure is horizontal, while that of trench gate structure is vertical. As the direction of MOS conductive channel changes, the corresponding internal junction capacitance changes. The comparative analysis of junction capacitance of two gate structures is also shown in Figure 1.

\footnotetext{
* Corresponding author: author@e-mail.org
} 


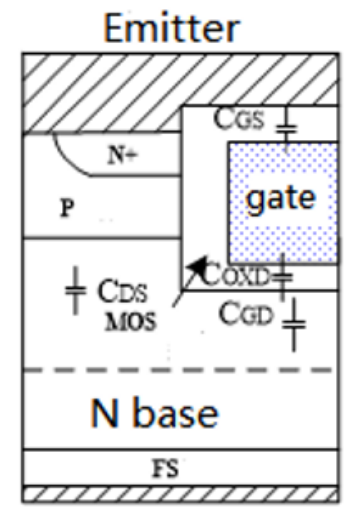

Collector

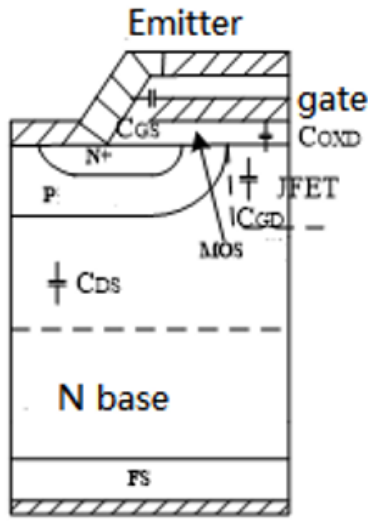

Collector
Fig1. Comparative analysis of junction capacitance of trench gate and planar gate

For planar gate structure, because the width of P-type body region is relatively small, it is considered that the width of depletion region covers the horizontal conductive channel, and the junction capacitance of gate-drain depletion layer of internal MOSFET can be expressed by a unified expression:

$$
C_{G D J}(t)=\frac{A_{G D} \varepsilon_{s i}}{\sqrt{2 \varepsilon_{s i}\left(V_{b c}(t)-V_{G S}(t)+V_{T d}\right) / q N_{L}}}
$$

In which: $A_{G D}$ is the lateral lateral area of trench gate deep into the base region; $\varepsilon_{s i}$ is the dielectric constant of silicon; $V_{b c}(t)$ is BJT base-emitter voltage drop; $V_{G S}(t)$ is the gate voltage; $V_{T d}$ is that threshold voltage of the gate-drain overlap depletion layer; $q$ is the electronic quantity; $N_{L}$ is the base doping concentration.

The corresponding gate-drain junction capacitance is expressed as:

$$
C_{G D}(t)=\left\{\begin{array}{l}
C_{O X D} \\
\frac{C_{O X D} C_{G D J}(t)}{C_{O X D}+C_{G D J}(t)}
\end{array}\right.
$$

However, for the trench gate structure, due to the large gate thickness, the width of the depletion layer may not cover the trench in IGBT switching transient, so it is necessary to analyze it according to different conditions.

\subsection{Analysis of trench gate junction capacitance}

In switching transient state, the internal current of IGBT includes the charge and discharge currents of gate capacitance and other internal junction capacitance besides hole current and electron current, which together constitute the total IGBT current. Therefore, the influence of capacitance must be taken into account in transient model analysis. The equivalent circuit diagram of IGBT including gate capacitance and internal junction capacitance is shown in Figure 2.

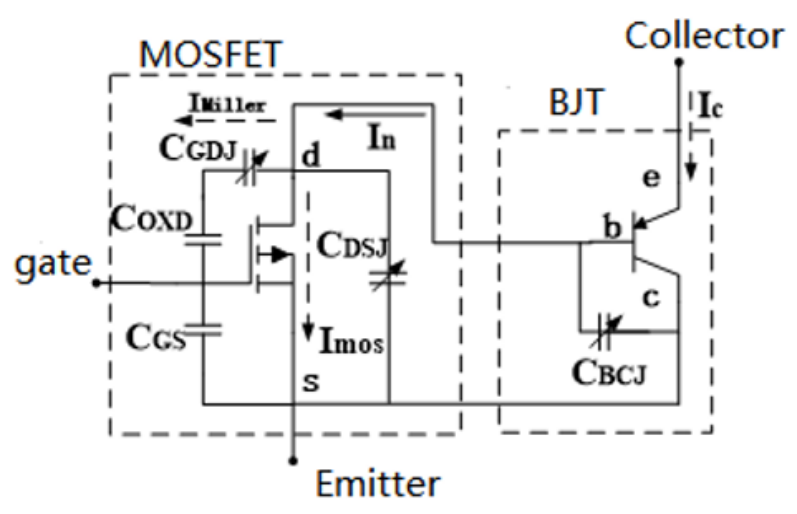

Fig2. IGBT equivalent circuit diagram

In Figure 2, $C_{G S}$ is the capacitance between gate and source; $C_{O X D}$ is the capacitance of grid overlapping oxide layer; $C_{G D J}$ is the grid overlapping depletion layer capacitance; $C_{O X D}$ and $C_{G D J}$ are connected in series to form the capacitance between gate and drain, that is $C_{G D}$, which is also feedback capacitance; $C_{D S J}$ is the capacitance between drain and source; $C_{B C J}$ is the junction capacitance between the $\mathrm{P}+$ emitter and the $\mathrm{N}$ base region, which can be approximately regarded as the sum of $C_{G D J}$ and $C_{D S J}$. Among them, the capacitors $C_{G S}$ and $C_{O X D}$ are all fixed values, while the capacitors $C_{G D J}, C_{D S J}$ and $C_{B C J}$ all change with the change of the width of the depletion layer.

According to the relationship between depletion layer width and trench gate width without considering trench gate structure, the junction capacitance of trench gate structure in Figure 2 is also analyzed in two cases.

(1) The depletion layer is not widened to cover the trench gate, that is $W_{d e p, P I N} \leq L_{x}$.

The internal junction capacitance of the trench gate IGBT is shown in Figure 3.

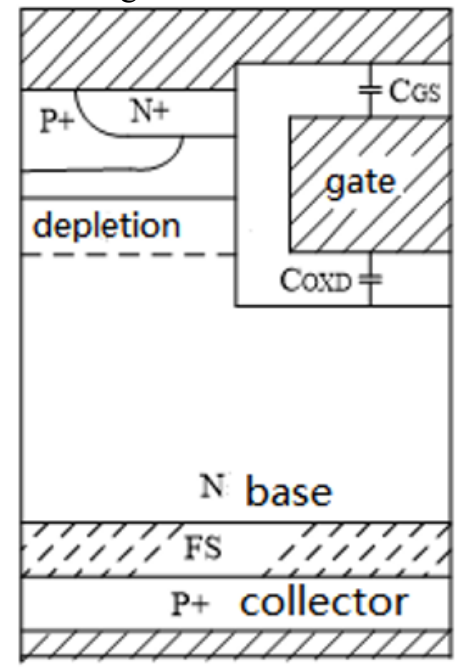

Fig3. Internal junction capacitance under condition 1

Because the depletion layer fails to widen and cover the trench gate, there is no depletion layer at the lower part of the trench gate, and the grid-drain junction capacitance 
only needs to consider the oxide layer capacitance at the lower part of the trench gate. At this time, the junction capacitance is expressed as:

$$
C_{G D}=C_{O X D}
$$

Where: $C_{O X D}$ represents the capacitance of oxide layer at the lower part of trench gate.

(2) The depletion layer can be widened to cover the trench gate, that is $W_{d e p, P I N} \leq L_{x}$. 4.

The internal junction capacitance is as shown in Figure

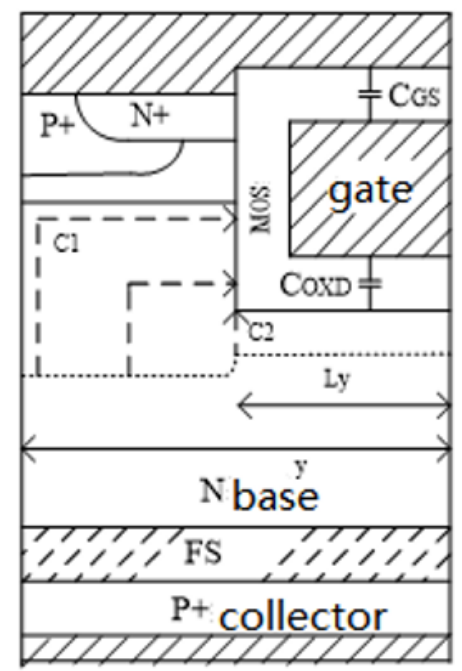

Fig4. Internal junction capacitance under condition 2

Because of carrier movement and current flow, it is necessary to consider the longitudinal depletion layer capacitance at the lower part of trench gate, the lateral depletion layer junction capacitance at the side, and the trench gate oxide layer capacitance.

The vertical depletion layer junction capacitance of the gate-drain can be expressed as:

$$
C_{G D J, Z}(t)=\frac{A_{G D} \varepsilon_{s i}}{\sqrt{2 \varepsilon_{s i}\left(V_{b c}(t)-V_{G S}(t)+V_{T d}\right) / q N_{L}}-L_{x}}
$$

Where: $L_{x}$ is the longitudinal depth of trench gate.

The lateral depletion layer junction capacitance of gate-drain is the average of $\mathrm{C} 1$ and $\mathrm{C} 2$ depletion layer capacitances.

$$
\begin{array}{r}
C_{2}(t)=\frac{A_{G D H} \varepsilon_{s i}}{\sqrt{2 \varepsilon_{s i} V_{b c}(t) / q N_{L}}-L_{x}} \\
C_{1}(t)=\frac{A_{G D H} \varepsilon_{s i}}{\sqrt{2 \varepsilon_{s i} V_{b c}(t) / q N_{L}}+y-L_{y}} \\
C_{G D J, H}(t)=\frac{C_{1}(t)+C_{2}(t)}{2}
\end{array}
$$

The grid-drain depletion layer junction capacitance is obtained by connecting the horizontal and vertical depletion layer capacitances in parallel

$$
C_{G D J}(t)=C_{G D J, Z}(t)+C_{G D J, H}(t)
$$

The gate-drain Miller capacitor is formed by connecting the gate-drain depletion layer capacitor and the oxide layer capacitor in series.

Combining the above two cases, the expression of gate-drain capacitance is obtained as follows:

$$
C_{G D}(t)= \begin{cases}C_{O X D} & W_{d e p, P I N} \leq L_{x} \\ \frac{C_{O X D} C_{G D J}(t)}{C_{O X D}+C_{G D J}(t)} & W_{d e p, P I N}>L_{x}\end{cases}
$$

Because the junction capacitance of depletion layer is affected by external voltage, when the voltage rises, the junction capacitance of depletion layer will drop rapidly. Therefore, in the linear region, the feedback capacitance is basically equal to the oxide layer capacitance, while in the cut-off region and saturation region, the feedback capacitance is basically equal to the depletion layer capacitance.

Gate-drain junction capacitance $C_{G D}(t)$, drainsource junction capacitance $C_{D S}(t)$ and base-collector junction capacitance $C_{B C}(t)$ are all variable junction capacitances, which have high capacitance in the linear region of low voltage and decrease with the increase of depletion layer width, i.e., applied voltage.

Three capacitors are usually given in IGBT manual, namely input capacitor $C_{i s s}$, output capacitor $C_{o s s}$ and feedback capacitor $C_{r s s}$. The relationship between these capacitors and the internal junction capacitance in Figure 2 is as follows:

$$
\left\{\begin{array}{c}
C_{i s s}=C_{G S}+C_{G D} \\
C_{r s s}=C_{G D} \\
C_{o s s}=C_{D S}+C_{G D}
\end{array}\right.
$$

The MOS channel current meets the following requirements according to the conditions that MOSFET works in cut-off region, linear region and saturation region:

$$
I_{\text {mos }}(t)=\left\{\begin{array}{l}
0 \\
K_{P}\left(V_{G S}(t)-V_{t h}\right) V_{d s}(t)-K_{P} V_{d s}^{2}(t) / 2 \\
K_{P}\left(V_{G S}(t)-V_{t h}\right)^{2} / 2
\end{array}\right.
$$

The expression of base current obtained from the equivalent circuit of fig. 2 is:

$$
\begin{aligned}
& I_{n L}(x=W)=I_{m o s}(t)+\left(C_{D S}+C_{G D}\right) \frac{d V_{d s}(t)}{d t} \\
& -C_{G D} \frac{d V_{G S}(t)}{d t}
\end{aligned}
$$

The expression of gate voltage change rate is:

$$
\frac{d V_{G S}(t)}{d t}=\frac{I_{G}(t)}{C_{G S}+C_{G D}(t)}+\frac{C_{G D}(t)}{C_{G S}+C_{G D}(t)} \frac{d V_{b c}(t)}{d t}(13)
$$

The expression of voltage change rate under different conditions can be obtained by introducing the expressions of gate-drain junction capacitance under two different conditions.

\section{Experimental verification}

A trench gate field termination IGBT module with KW60N65S model is selected, and the rated voltage and current levels are $650 \mathrm{~V}$ and $60 \mathrm{~A}$ respectively. The experimental test conditions are as follows: the bus voltage is set to $400 \mathrm{~V}$, the driving voltage is $15 \mathrm{~V}$ when 
switching on, and the load inductance is $500 \mathrm{uH}$. The collector-generator voltage and collector current of IGBT are measured by double pulse test method. As the accurate calculation of junction capacitance mainly affects the turnoff current in IGBT transient process, the turn-off current is compared. The test circuit is shown in Figure 5.

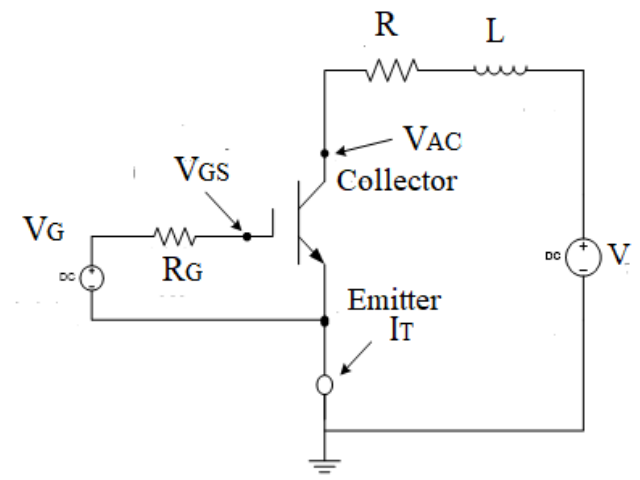

Fig5. Experimental test circuit

Using the junction capacitance calculation method of planar gate structure, the simulation waveform of IGBT turn-off transient current and the comparison of experimental waveforms are shown in Figure6.

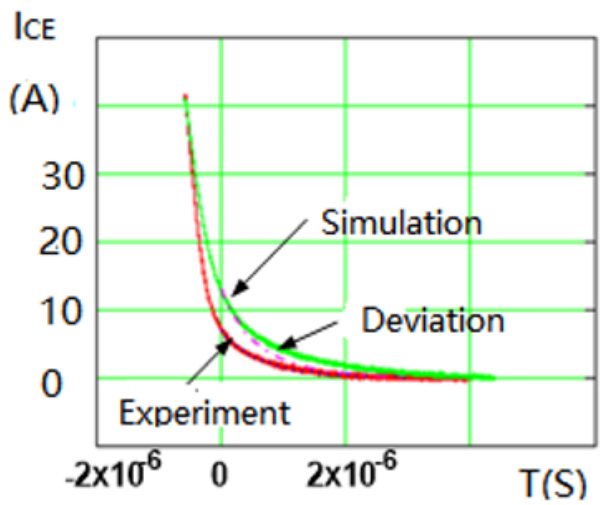

Fig6. Comparison of shutdown currents

Using the calculation method of junction capacitance in this paper, the simulation waveform of IGBT turn-off transient current and the comparison of experimental waveform are shown in Figure 7.

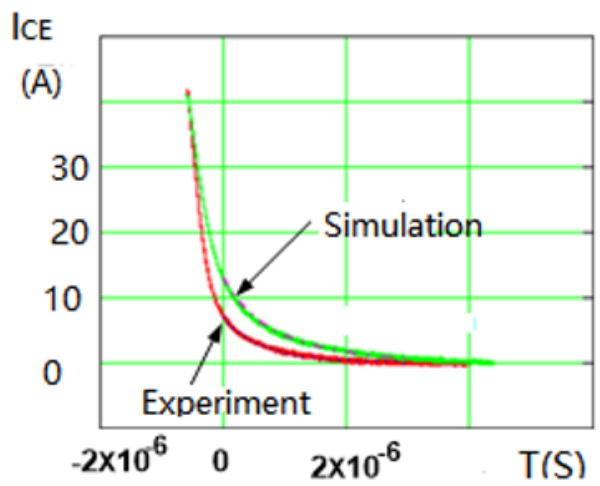

Fig7. Comparison of shutdown currents

It can be seen from Figure 6 and Figure 7 that the simulated turn-off transient current obtained by the calculation method of junction capacitance in this paper is closer to the experimental waveform.

\section{Summary}

According to the difference between the traditional planar gate structure and the new trench gate structure, this paper discusses the calculation method of junction capacitance in IGBT in two cases according to whether the depletion layer in the base region can widen and completely cover the trench gate, which overcomes the single junction capacitance treatment method in planar gate structure.

\section{Acknowledgments}

This work was supported by the 2018 year Youth Foundation of Wuhan Donghu University(2018dhzk005), and the Scientific Research Project of Hubei Education Department in 2019(B2019267).

\section{References}

1. Chen Xudong, Cheng Jianbing, Teng Guobing, et al. Novel trench gate field stop IGBT with trench shorted anode[J]. Iournal of Semiconductors, 2016, 37(5): 6164.

2. Bazzi A, Philip T, Jonathan W. IGBT and diode loss estimation under hysteresis switching[J]. IEEE Transactions on Power Eletronics, 2012, 27(3): 10441048.

3. Chamund D J, Coulbeck L, Newcombe D R, et al . High PowerDensity IGBT Module for High Reliability Application[C]. Proceeding of IPEMC, 2009: 274-280.

4. Hu Shao-wei, Zhu Yang-jun, Duan Yao-yu. An Impact Analysis of Gate Resistance on Static and Dynamic Dissipation of IGBT Modules[C]. Proceeding of ICECC, 2011:715-718.

5. Vinod Kumar Khanna, The Insulated Gate Bipolar Transistor-IGBT Theory and Dedign[J].IEEE Society Press. 2003:105-107. 